\title{
Validation of AIRS/AMSU cloud retrievals using MODIS cloud analyses
}

\author{
Gyula I. Molnar and Joel Susskind \\ UMBC/JCET \& NASA GSFC, Code 613, Greenbelt, MD 20771
}

\begin{abstract}
The AIRS/AMSU (flying on the EOS-AQUA satellite) sounding retrieval methodology allows for the retrieval of key atmospheric/surface parameters under partially cloudy conditions (Susskind et al. ${ }^{1}$ ). In addition, cloud parameters are also derived from the AIRS/AMSU observations. Within each AIRS footprint, cloud parameters at up to 2 cloud layers are determined with differing cloud top pressures and "effective" (product of infrared emissivity at $11 \mu \mathrm{m}$ and physical cloud fraction) cloud fractions. However, so far the AIRS cloud product has not been rigorously evaluated/validated.

Fortunately, collocated/coincident radiances measured by MODIS/AQUA (at a much lower spectral resolution but roughly an order of-magnitude higher spatial resolution than that of AIRS) are used to determine analogous cloud products from MODIS. This allows us for a rather rare and interesting possibility: the intercomparisons and mutual validation of imager vs. sounder-based cloud products obtained from the same satellite positions.

First, we present results of small-scale (granules) instantaneous intercomparisons. Next, we will evaluate differences of temporally averaged (monthly) means as well as the representation of inter-annual variability of cloud parameters as presented by the two cloud data sets. In particular, we present statistical differences in the retrieved parameters of cloud fraction and cloud top pressure. We will investigate what type of cloud systems are retrieved most consistently (if any) with both retrieval schemes, and attempt to assess reasons behind statistically significant differences.
\end{abstract}

Keywords: infrared, remote sensing, clouds, cloud top pressure, effective cloud fraction, inter-annual variability, global climatology

\section{INTRODUCTION}

Clouds appear to play an important role in the atmosphere on all spatial and temporal (weather to long-term climate changes) scales. However, our knowledge of their behavior, in particular their interaction with climate changes, is still lacking. Satellite can provide an ideal platform to access cloud and large scale atmospheric variables simultaneously and on comparable scales. This is extremely important for developing more reliable cloud models, which could help to improve the representation of cloud-climate feedbacks (probably the least known ones, still hindering global warming predictions, for example) in global general circulation models. The TOVS satellites were the first to provide this information since 1979 on. For example, the ISCCP cloud project is using TOVS-derived atmospheric temperature profiles to better their product (Zhang et al., ${ }^{2}$ ). Susskind et al., ${ }^{3}$ also have generated cloud fields using TOVS observations. The AIRS/AMSU can accomplish the same tasks with a much higher accuracy (Pagano et al. $\left.{ }^{4}\right)$. It would be very useful for climate change assessments to have the longest possible satellite cloud climatology, so beside this AIRS work, we also plan to revisit/validate the TOVS-based cloud retrieval results. In addition, we also plan to assess (probably) one of the most important parameters of global energy balance namely the outgoing longwave radiation or OLR. Of course, proper representation/retrieval of clouds is very important in this context; whatever cloud distribution a retrieval scheme provides, must be consistent with energy balance, $i$. e., with the observed OLR values (restricting ourselves to the Earth's thermal radiation for now-solar/shortwave energy balance and the retrieval of associated cloud characteristics is an even more difficult issue). We believe, part of the discrepancies between AIRS and MODIS cloud fields, to be shown in this work, may be related to the "non-energy balance" nature of threshold-based cloud fraction (cloud mask) retrievals by MODIS (cf., King et al., ${ }^{5}$ ). 
The AIRS/AMSU (flying on the EOS-AQUA satellite) sounding retrieval methodology allows for the retrieval of key atmospheric/surface parameters under partially cloudy conditions (Susskind et al., ${ }^{1}$ ). In addition, cloud parameters are also derived from the AIRS/AMSU observations. Within each AIRS footprint, cloud parameters at up to 2 cloud layers are determined with differing cloud top pressures and "effective" cloud fractions. However, so far the AIRS cloud product has not been rigorously evaluated/validated.

Why compare AIRS clouds with MODIS cloud retrievals? AIRS is on the same satellite as MODIS-Aqua. Thus, for granule-scale intercomparisons, we can make sure that exactly the same scenes are being evaluated. However, for monthly mean cloud parameter intercomparisons MODIS-Terra cloud products (as computed directly by the MODIS team) were used, since they seem to be more reliable than those derived from the official gridded monthly mean MODIS-Aqua data. For Aqua, combining physical MODIS cloud cover and 11 $\mu \mathrm{m}$ cloud emissivities to obtain "effective cloud fraction" ( $\alpha \varepsilon$, where here $\alpha$ is geometrical fractional cloud cover and $\varepsilon$ is $11 \mu \mathrm{m}$ emissivity) which is the product most consistent with the AIRS cloud fraction, can be done only approximately using monthly means.

The following bullets highlight an overview of the AIRS/MSU retrieval methodology:

-Physically-based system;

-Independent of GCM except for surface pressure;

-Uses cloud cleared radiances to produce solution;

-Basic steps:

1) Microwave product parameters - solution agrees with AMSU-A radiances;

2) Initial cloud clearing using microwave product;

3) AIRS regression guess parameters based on cloud cleared radiances;

4) Update cloud clearing using AIRS regression guess parameters;

5) Sequentially determine surface parameters, temperature, moisture, ozone, $\mathrm{CO}$, and $\mathrm{CH}_{4}$ profiles;

-Apply quality control:

a) Select retrieved state - coupled AIRS/AMSU or AMSU only retrieval parameters;

b) Determine cloud parameters consistent with retrieved state and observed radiances;

c) Compute OLR, CLR sky OLR from all parameters via radiative transfer.

AIRS/AMSU CLOUD PRODUCTS: One set per Field-of-View of effective cloud fraction ( $\left.A_{\text {eff }}\right)$ for up to two cloud layers, as well as cloud top pressure $\left(\mathrm{C}_{\mathrm{tp}}\right)$ for up to two cloud layers.

\section{RESULTS}

First, we have performed collocated instantaneous retrieval intercomparisons using AIRS and MODIS measurements from the Aqua satellite. We have selected a mostly cloudy Granule (\#60) from AIRS Focus Day 09/06/02, centered above the Korean Peninsula, where most cloud types were present. Figs. $1 \mathrm{a}-1 \mathrm{~b}$ and $2 \mathrm{a}-2 \mathrm{~b}$ compare $A_{e f f}$ and $C_{t p}$ values averaged over $1^{\circ}$ bins for collocated grids of the AIRS and MODIS granules, respectively. Clearly, the MODIS cloud fractions are generally higher (much so for low-level clouds), whilst the AIRS clouds are generally at higher levels. 


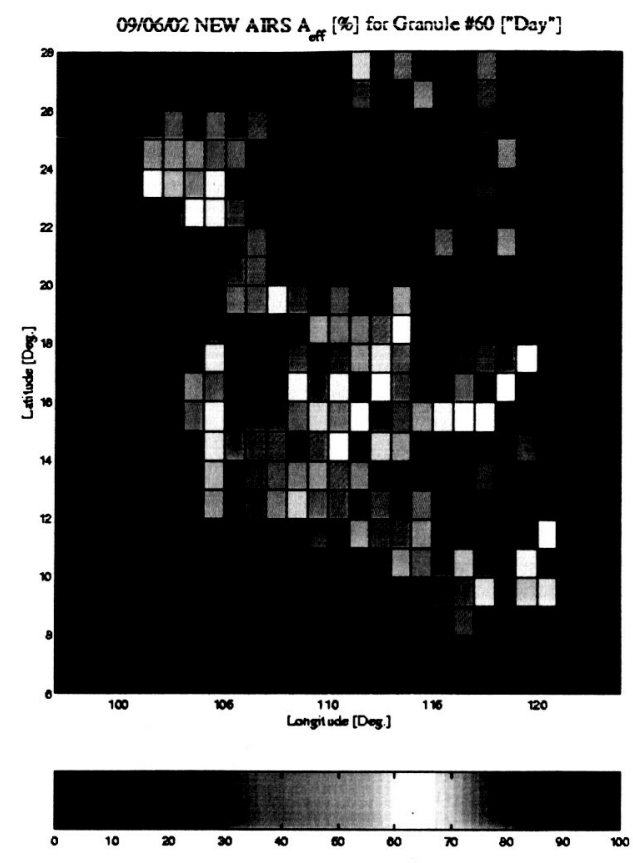

Figure 1a

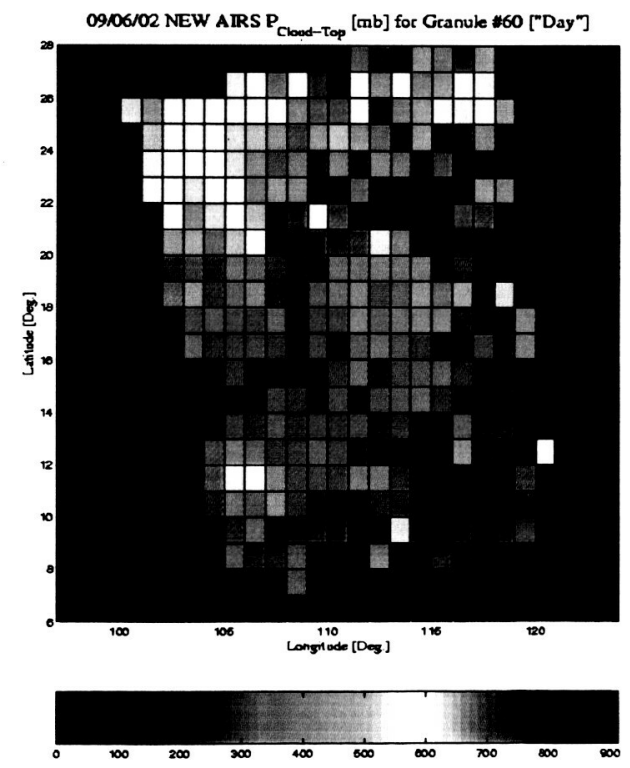

Figure 2a

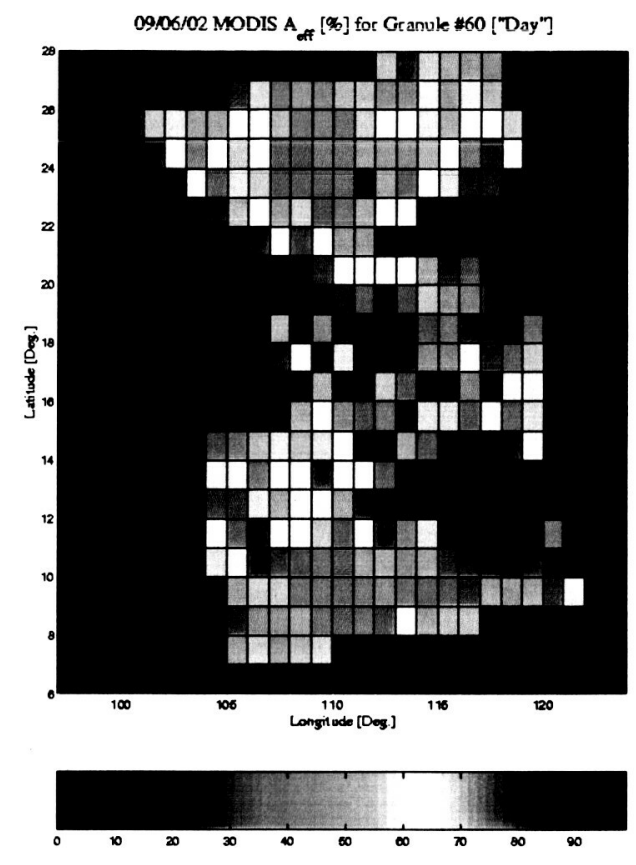

Figure $1 b$

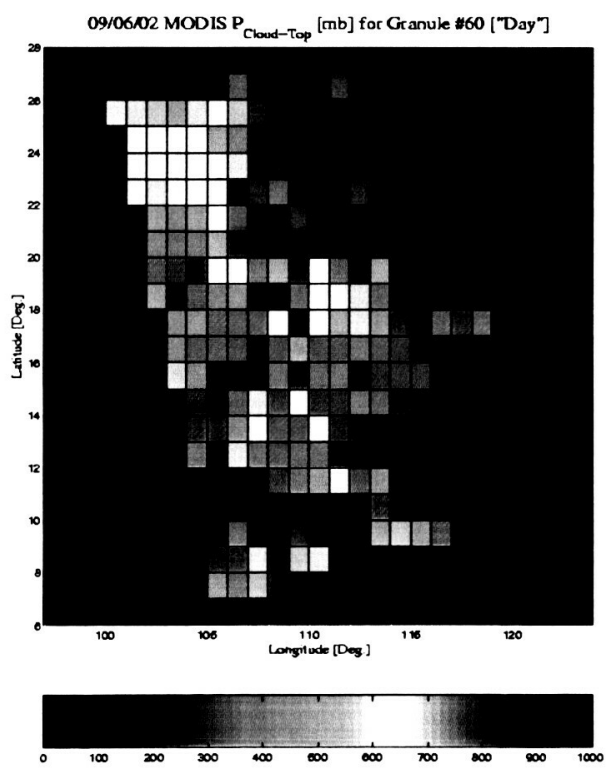

Figure $2 \mathrm{~b}$ 
Next, we have used monthly mean retrieval results to assess applicability of these cloud retrievals for climate



Figure 3
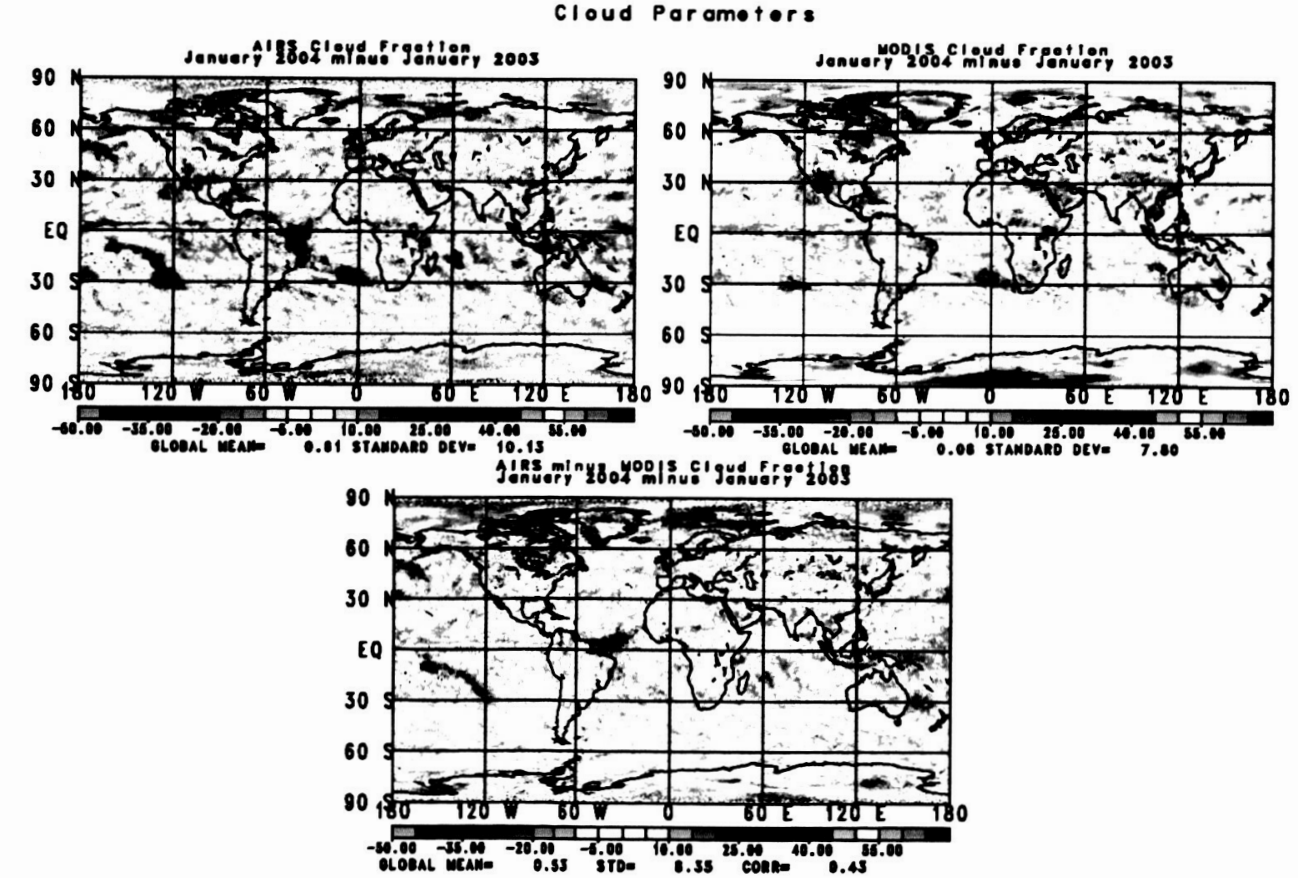

Figure 4 
variability assessment purposes. Fig. 3 illustrates AIRS-MODIS Jan./2003 and Jan./2004 cloud field intercomparisons in "combined" (both $\mathrm{A}_{\mathrm{eff}}$ and $\mathrm{C}_{\mathrm{tp}}$ are depicted concurrently) plots, whilst Fig. 4 shows the inter-annual variabilities as seen by each instrument, as well as the differences between the two retrieval results. Clouds are shown for 7 bins in terms of cloud top pressure (bin borders are shown above the color bars), and within each bin darker color shades indicate increasing cloud fractions in 5 bins of $20 \%$ cloud fraction range in each.

Note the general correspondence of the patterns, despite the obvious biases (AIRS mean cloud fraction is $\sim 43 \%$ vs. $\sim 67 \%$ of MODIS, whilst AIRS mean cloud top pressure is $\sim 520 \mathrm{hPa}$ vs. $\sim 670 \mathrm{hPa}$ in both Januaries). On the other hand, the inter-annual variabilities show quite a reassuring correspondence, meaning (potentially) that climatic trend-recognition could be close to identical for both retrieval schemes. The much larger intraseasonal differences are illustrated on Fig. 5, showing Aug./2004 vs. Jan./2004 clouds fields and their differences. Again, despite significant biases the intra-seasonal differences are quite close.
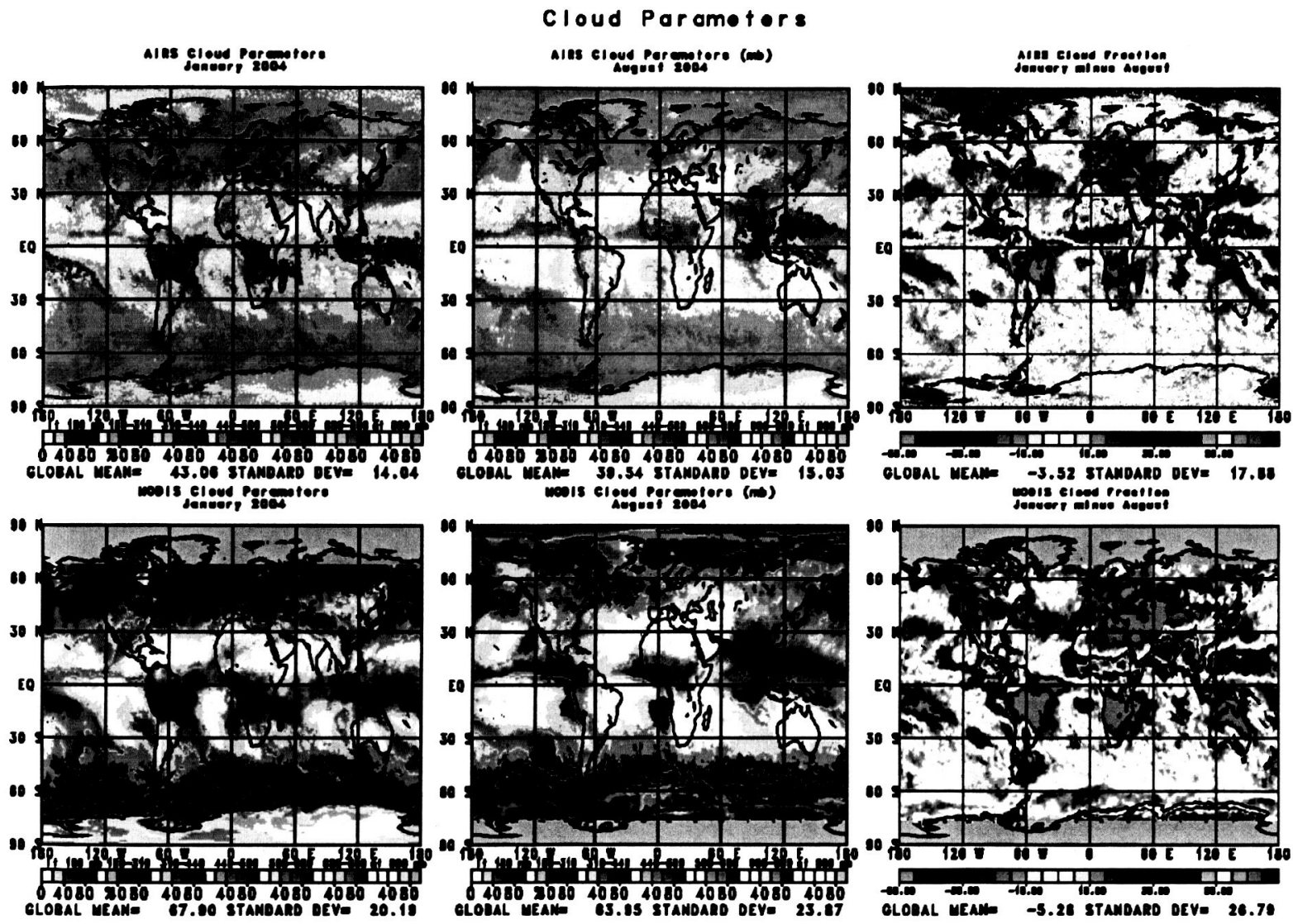

Figure 5

What is the latitudinal variability of these parameters? The following figures (Figs. 6a through $8 \mathrm{~b}$ ) illustrate the (in perhaps more easily digestible simple 2-D plots) latitudinal distribution of these cloud characteristics as well as their inter-annual, intra-seasonal and intra-instrument variabilities. In Figs. 9a-b, we have also paid attention to the separate evaluation of the 3 basic cloud types using the same criteria as ISCCP (in terms of cloud top height). Clouds were separated to low- (1000-680 hPa), middle- (440-680 hPA), and high (<440 hPa) level clouds. 

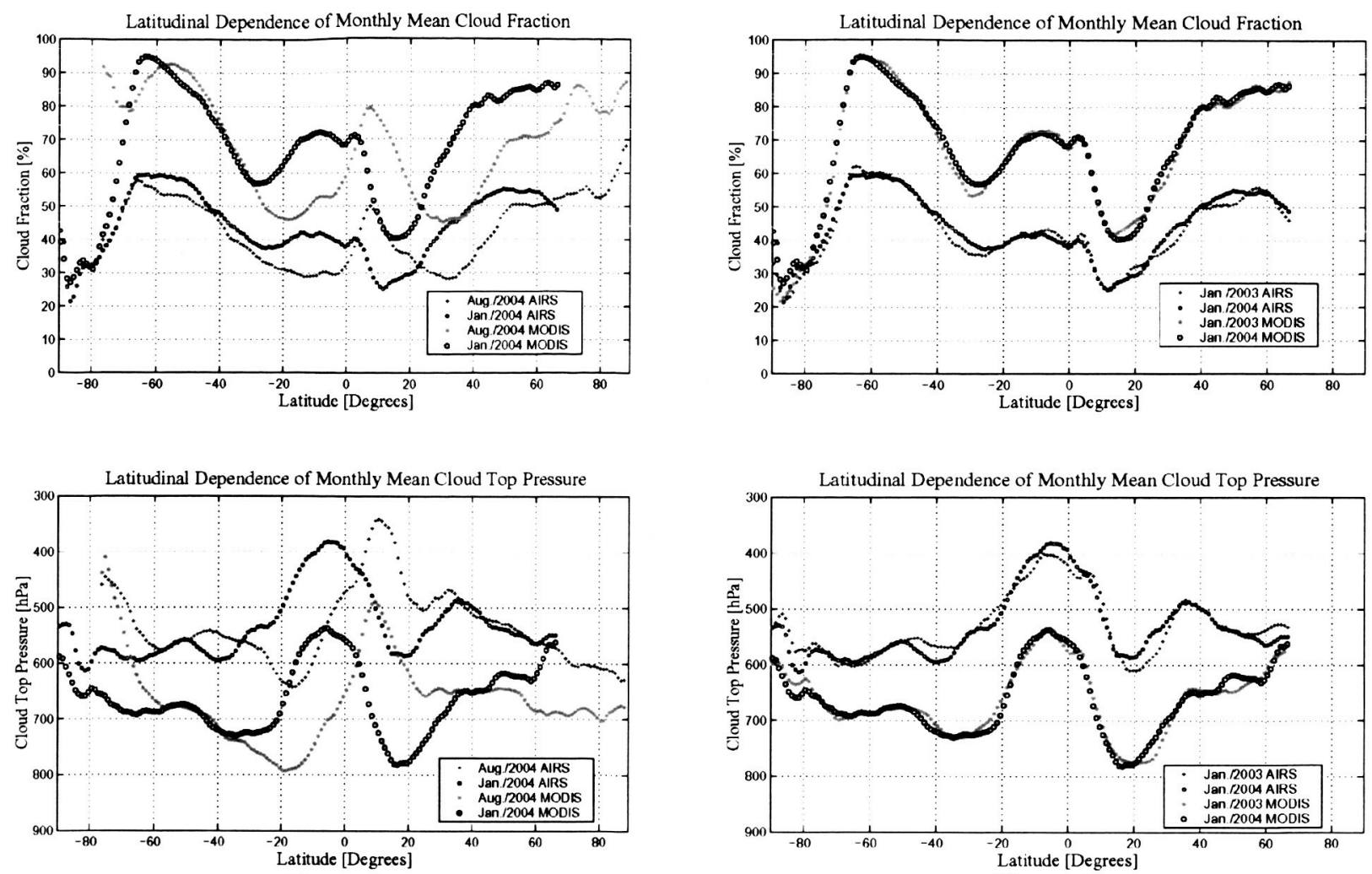

Figure 6a
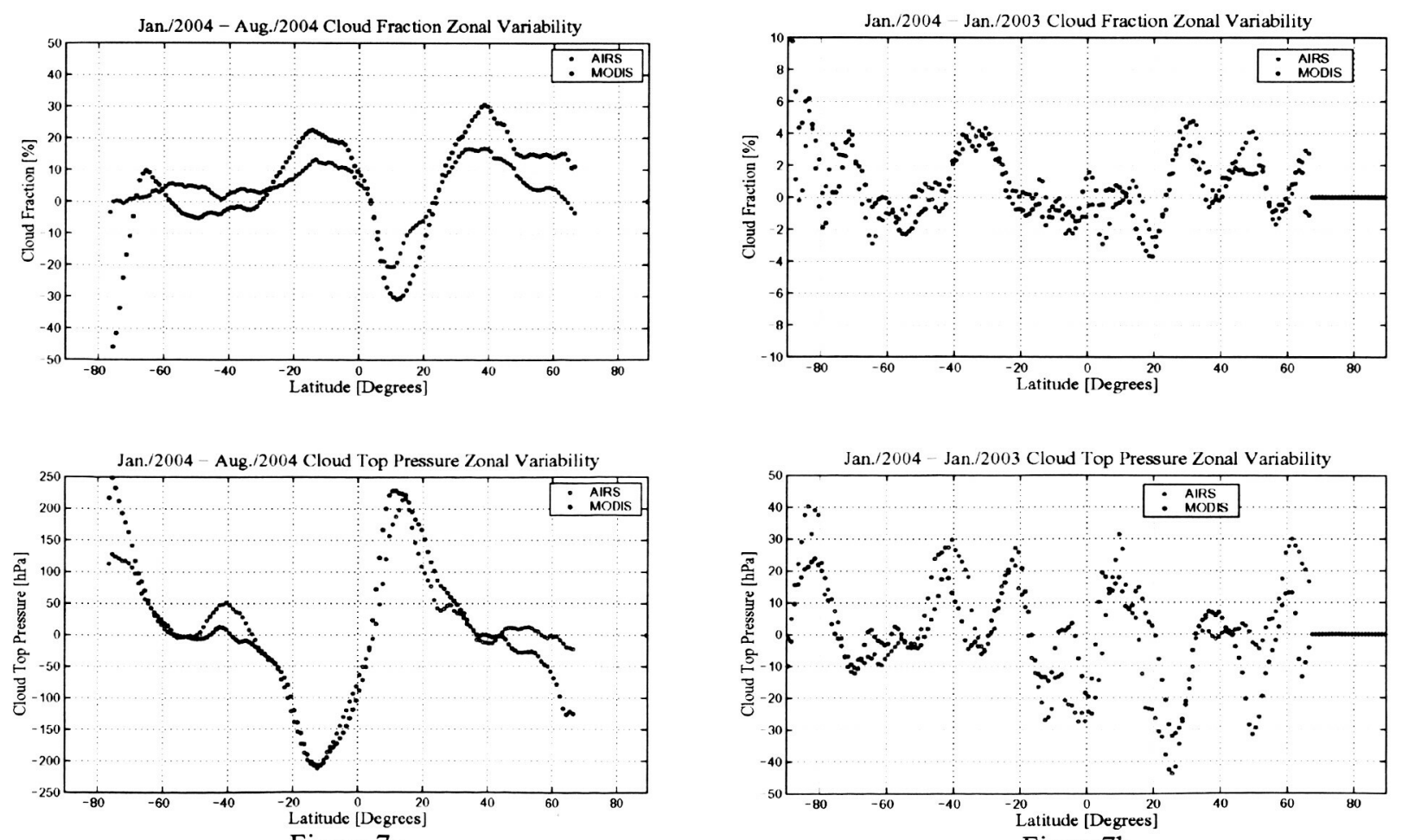

Figure 7a

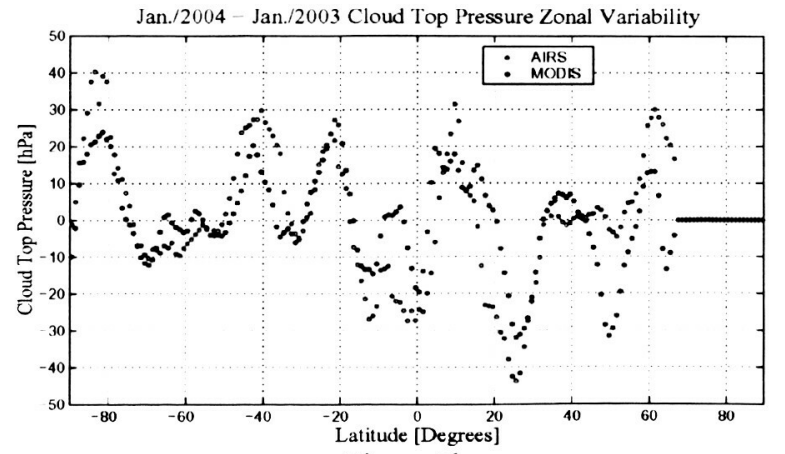

Figure $7 b$ 

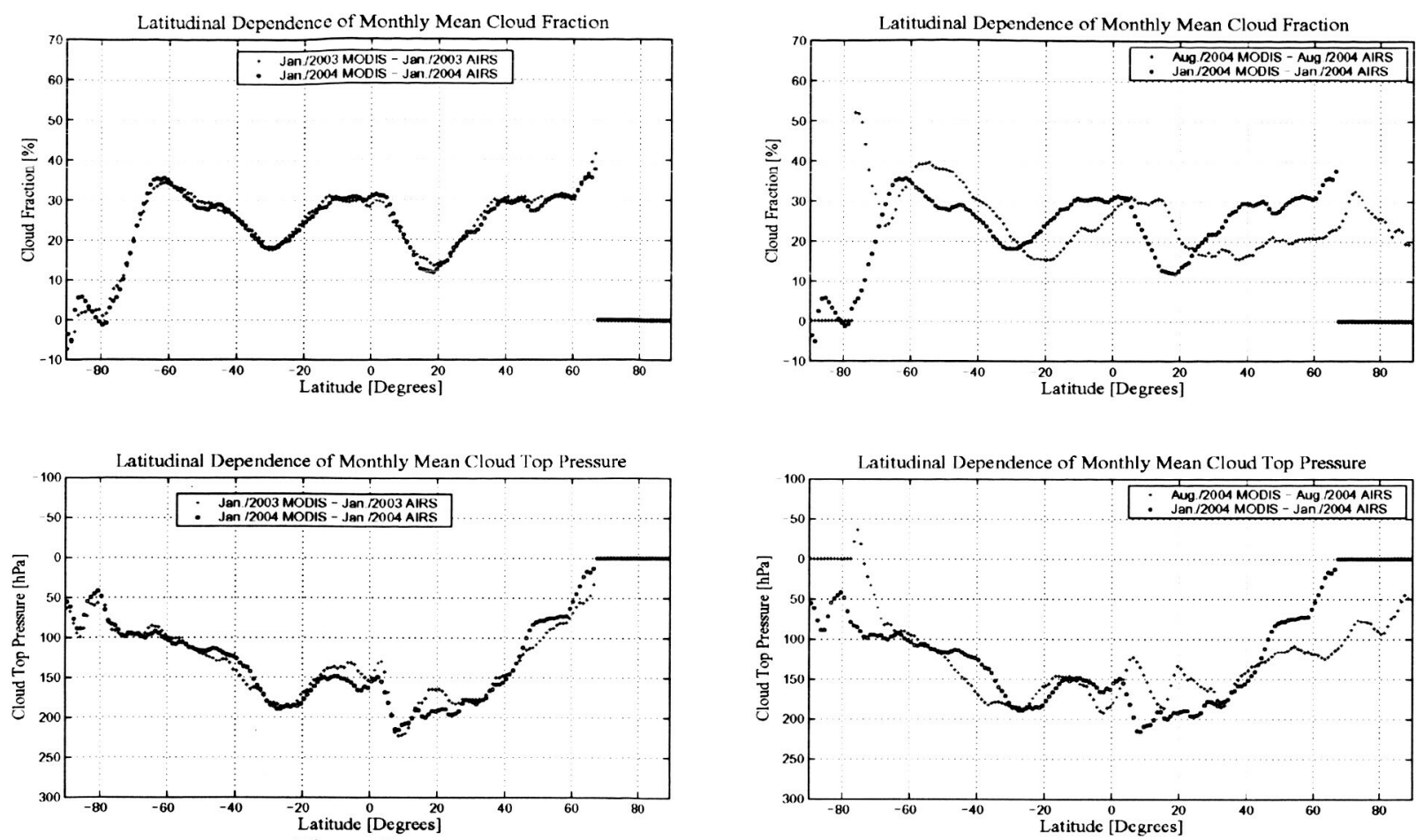

Figure 8a

Figure $8 \mathrm{~b}$

Finally, Figs. 9a-c illustrate the latitudinal averages of Low-, Middle- and High cloud parameters, respectively. Clearly, the great discrepancy of the low cloud fraction in particular cries out for explanation first.

AIRS high clouds are more extensive, middle cloud distributions are very different in the Tropics. Note that for all cloud type, almost all the time, the AIRS clouds are at higher levels (lower cloud top pressures). It seems kind of surprising that the overall parallel shape (see Figs. 6a through 8b), of the average latitudinal total cloudiness parameters breaks down when individual cloud layers are considered.
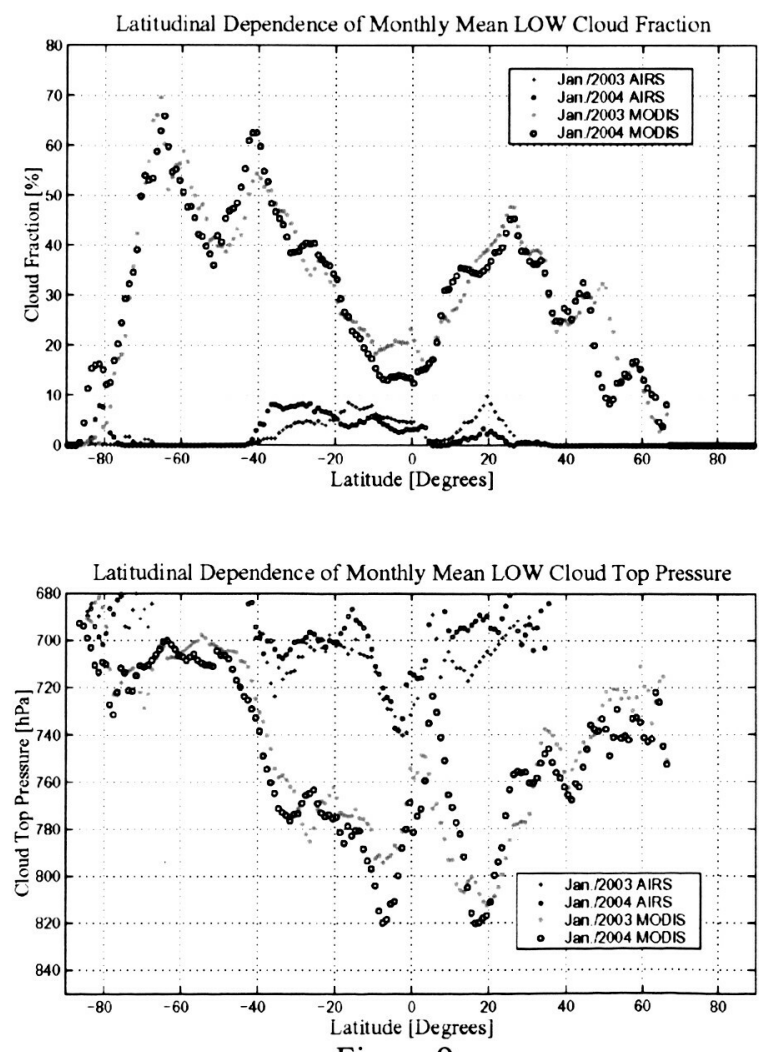

Figure 9a 

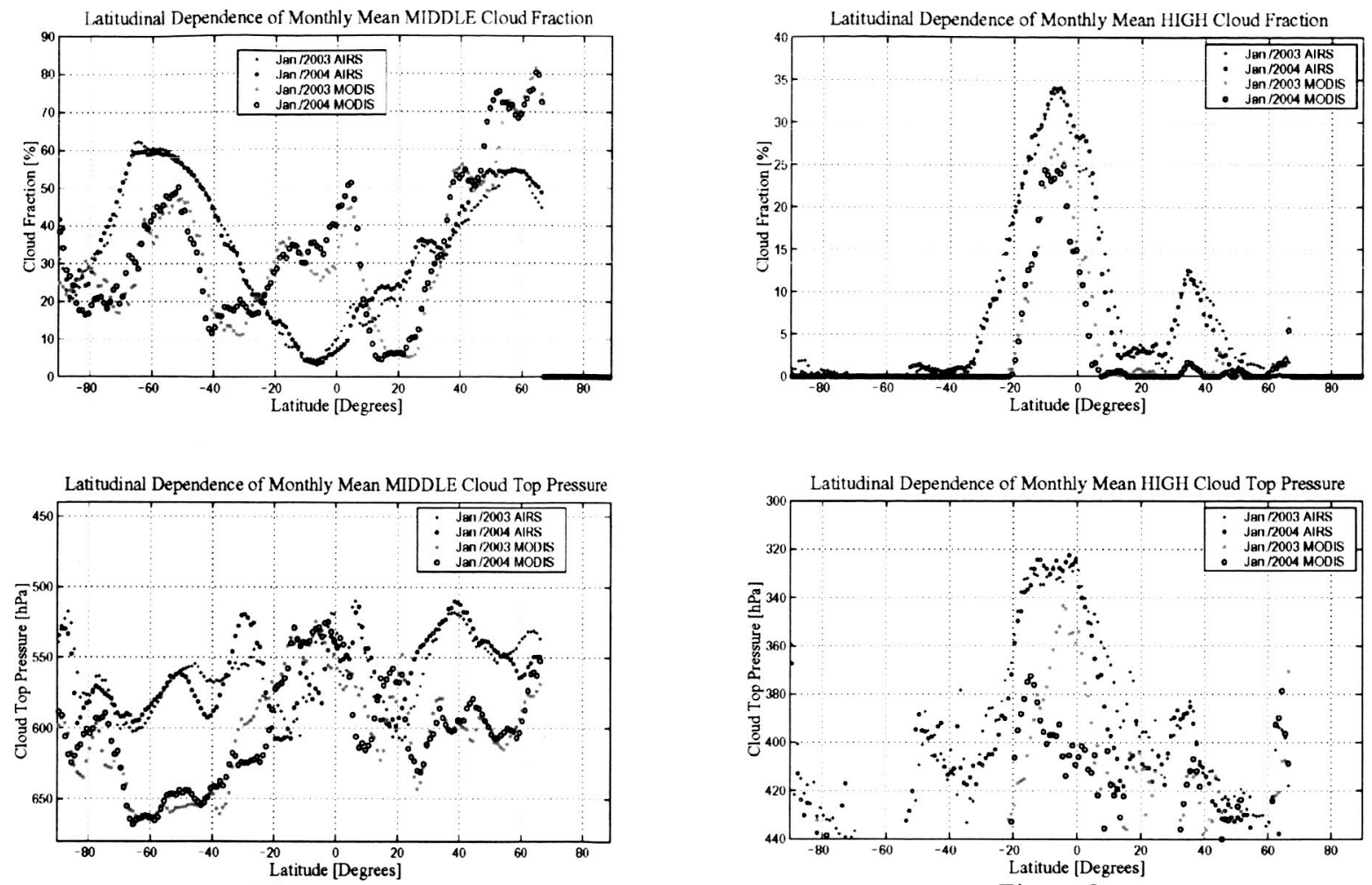

Figure 9a

Figure 9c

\section{DISCUSSION}

What could be main reasons for the significant differences? Our preliminary assessment can be summarized in the following remarks:

1) The AIRS cloud-clearing is essentially an "energy-balance" approach, i. e., the "observed" - "computed" radiance errors are minimized with the retrieved cloud distribution. This is also clearly illustrated by the excellent correspondence between AIRS and CERES OLR values obtained in our ongoing parallel study to be reported later in detail. Here we just present Figs. 10-11 to illustrate the excellent correspondence between monthly mean OLR values as well as between their variabilities.

2) On the other hand, the MODIS cloud-mask is essentially (multi)-threshold based. This, by itself could explain a significant positive cloud fraction bias even "reasonable" threshold values may lead to misidentification of partially cloudy pixels (PCPs) as 'cloudy', much more often than 'clear'. The main reason for this "bias" is that currently operational fixed threshold techniques ( $c f$. Rossow et al., ${ }^{5}$ ) treat individual pixels as being either overcast or cloud-free. This assumption may lead to biases in the retrieved fractional cloud cover because:

i) The retrieved cloud fraction is sensitive to the choice of threshold. Moreover, if a threshold was chosen to balance out the errors in mean cloud cover for one cloud type, then biases would appear for other cloud types. Furthermore, using a fixed threshold also makes the retrieved fractional cloud cover sensitive to instrument calibration errors. This is especially a problem when we want to create the longest possible satellite cloud climatology, necessary for global change assessments.

ii) The cloud cover is sensitive to data-resolution (i. e., pixel size) since errors in fractional cloudiness derived by using a fixed threshold depend not only on the cloud cover but also on cloud size (e. g., Coakley and Baldwin $^{6}$, Coakley ${ }^{7}$, Wielicki and Parker ${ }^{8}$, Harshvardhan et al., ${ }^{9}$ ). In general, the larger the pixel size, the less accurate the results because the greater likelihood that the pixel is neither cloud-free nor completely cloudcovered. 
Outgolng Longware Radlotion (wotts/m
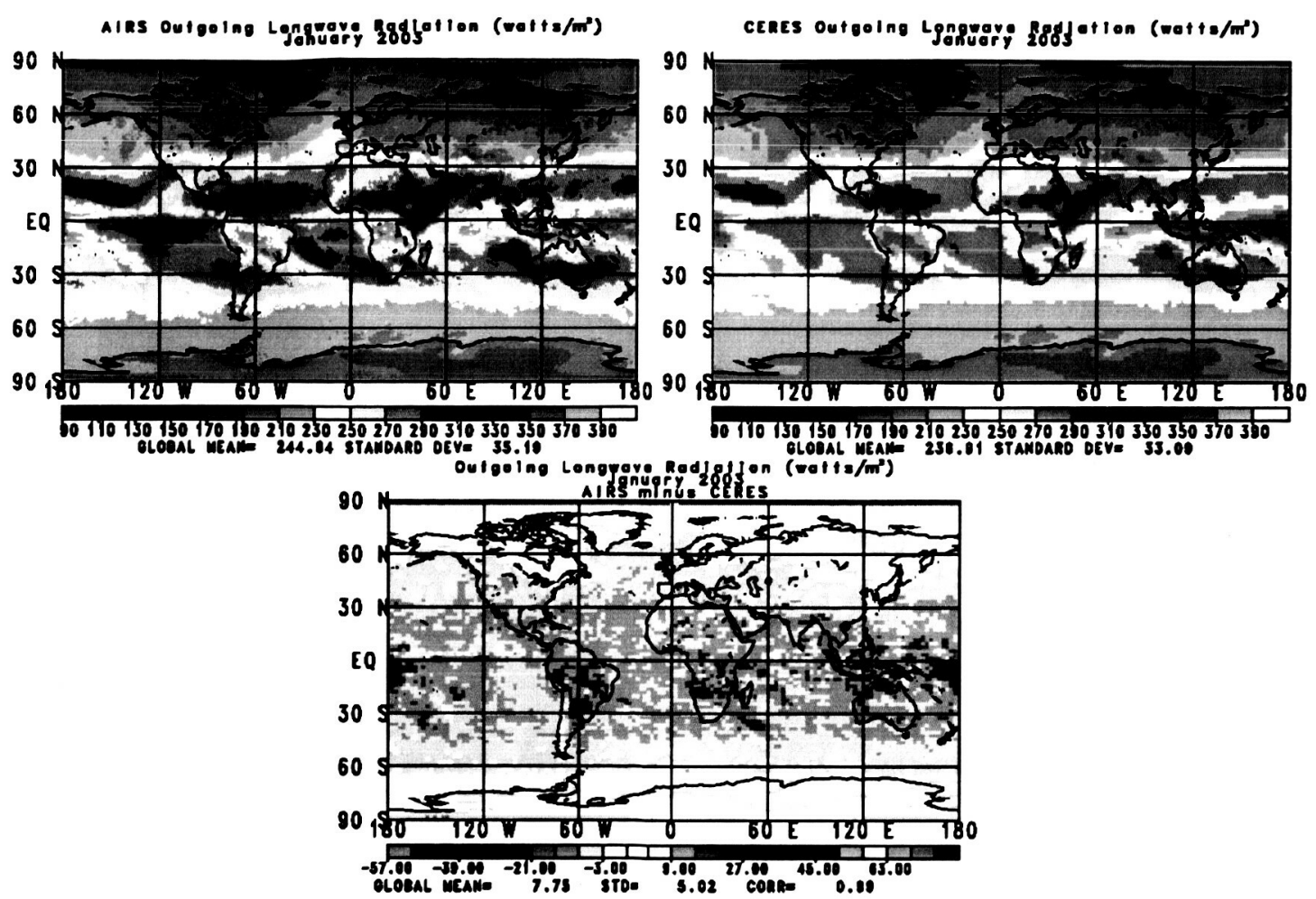

Figure 10

Outgolng Longware Radlation (wotts/m
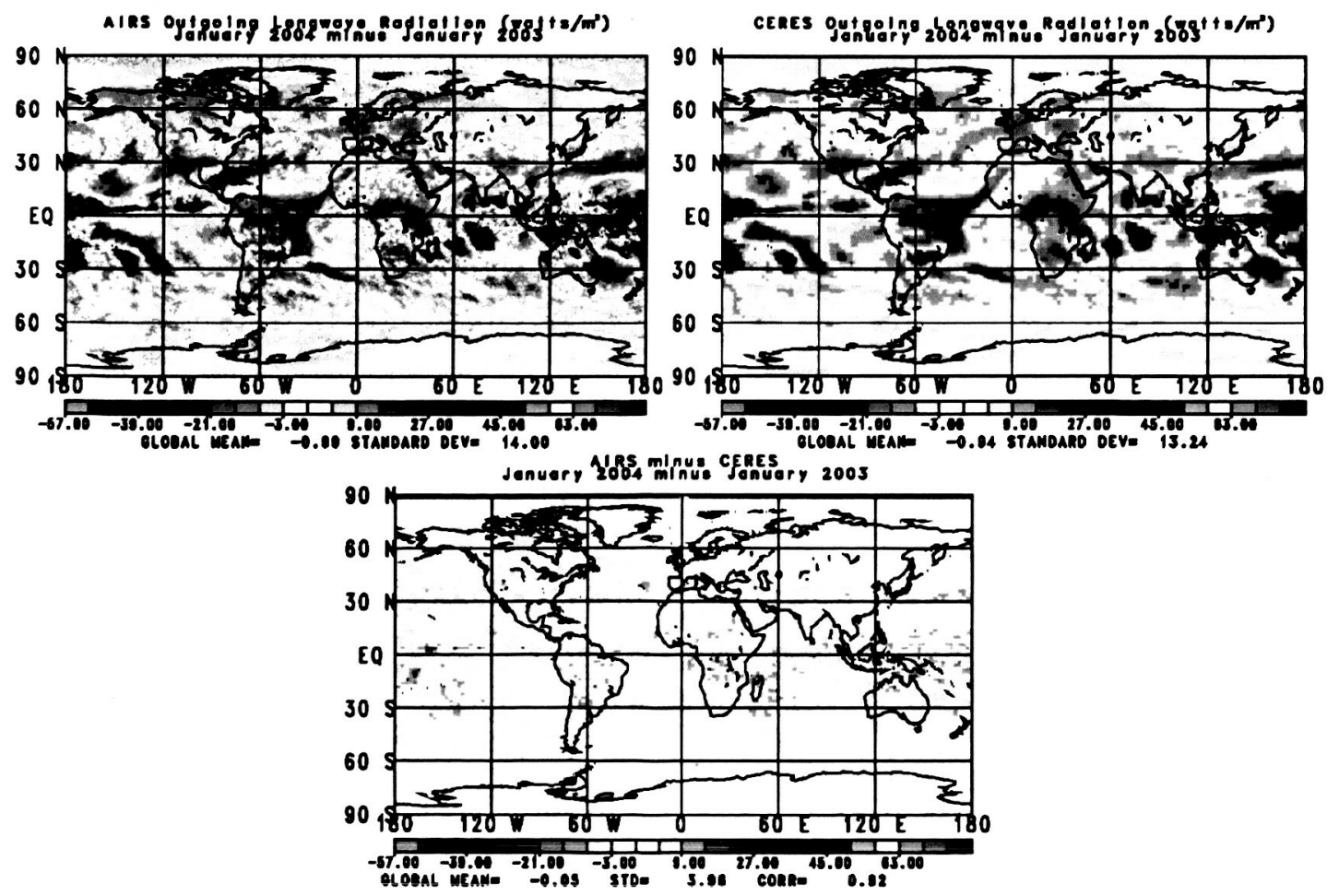

Figure 11 
Both problems could be related to the quite common presence of partially cloudy pixels (PCPs) as demonstrated for example by Coakley and Bretherton ${ }^{10}$, Coakley ${ }^{7}$, as well as Chang and Coakley ${ }^{11}$. For the sake of further illustrating this point, let us use a fixed threshold technique (referred to as TH for brevity in the followings) to evaluate cloud fraction for an infrared window (IR) satellite image scene where the cloud elements are subresolution. For the sake of simplicity, let us simulate a small oceanic scene of $32 \times 32$ pixels, corresponding to a $\sim(32 \mathrm{~km})^{2}$ size, $1 \mathrm{~km}$ nominal resolution MODIS infrared (IR) $11 \mu \mathrm{m}$ window channel image where the cloud elements are optically thick at the wavelength of the observation and they reside in a well-defined layer with cloud top radiance of $80 \mathrm{~mW} \mathrm{~m}^{-2} \mathrm{SR}^{-1} \mathrm{~cm}$ (corresponding to an approximate brightness temperature (B) $\mathrm{T}$ of $279.7 \mathrm{~K}$ in the window channel). At the same time, let the ocean surface have a uniform sea surface temperature (SST) corresponding to $100 \mathrm{mWm}^{-2} \mathrm{SR}^{-1} \mathrm{~cm}$ surface upwelling radiance $(i . e$, the corresponding BT is $\sim 293.1 \mathrm{~K}$ ) as measured by the satellite sensor. Note that these values are quite representative for marine stratiform cloud systems, the cloud top of which is being capped by the marine boundary layer. The prevailing subresolution cloudiness is represented by randomly varying pixel radiances in the $80-100 \mathrm{mWm}^{-2} \mathrm{SR}^{-1} \mathrm{~cm}$ interval. Fig. 12 shows the corresponding simulated (smoothed) "satellite image" in terms of ${ }^{\circ} \mathrm{C}$ BT in order to make threshold selection easier. Applying the commonly used SST-2.5 ${ }^{\circ} \mathrm{C}$ threshold, the $17.3^{\circ} \mathrm{C}$ contour is also shown. It is obvious that the fractional cloud amount corresponding to this threshold is far greater than the real 0.50 value, 0.80 , in fact. Furthermore, for such a small scene (with less inherent SST variability) an SST $-1.0^{\circ} \mathrm{C}$ threshold is probably a more reasonable assumption; applying this, the TH-derived cloud fraction rises to 0.91 .

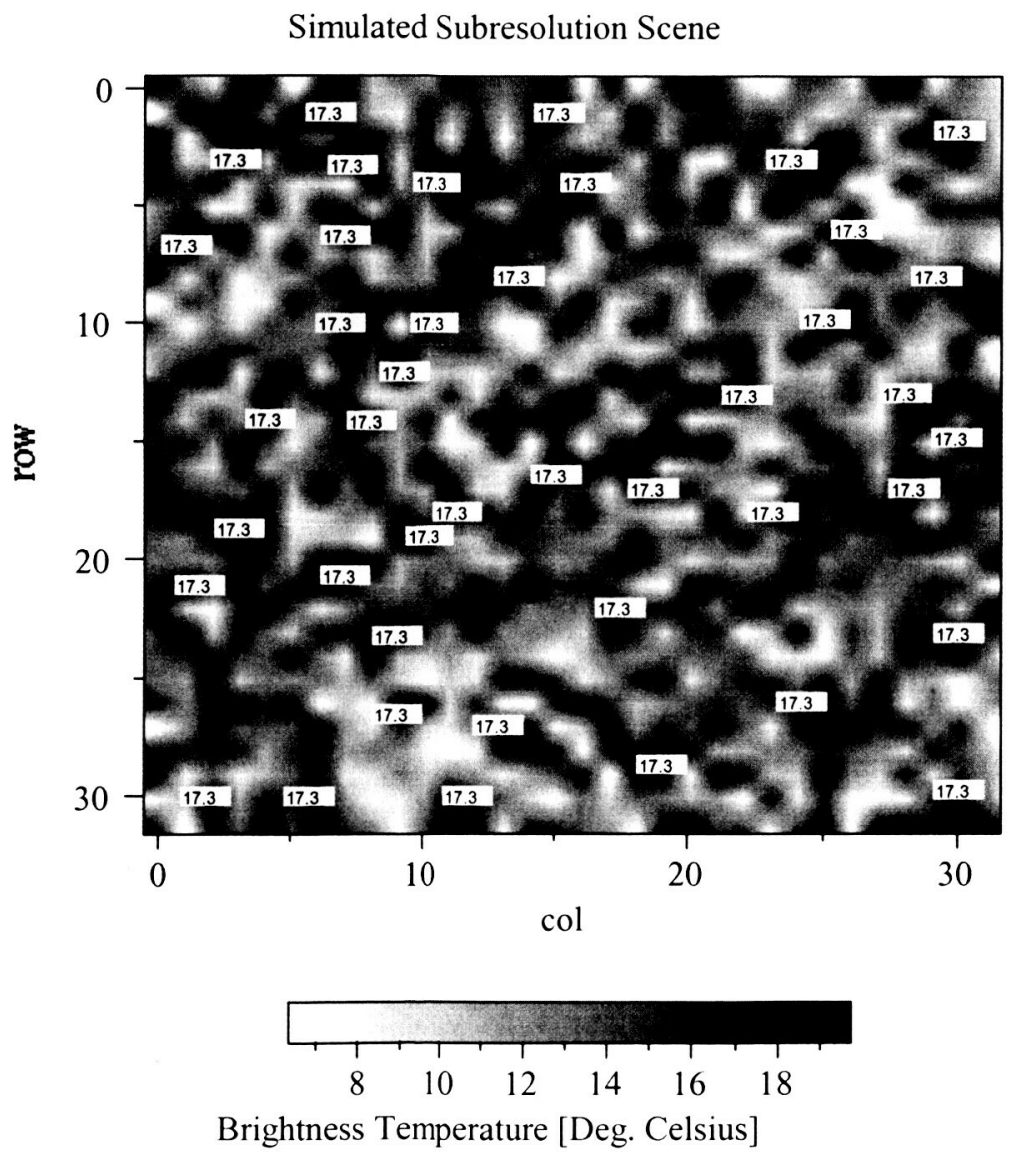

Figure 12

Thus, AIRS cloud fraction is expected to be lower than that of MODIS because the MODIS low cloud cover is expected to have a rather strong positive bias due to the prevalence of the above-discussed PCPs in low level cloud systems, like marine stratus. 
Furthermore, there could be another/additional causes of this discrepancy: effects of semitransparent (in the IR window channels) cloud elements could also be the (partial) cause. Because the underlying surface is generally warmer than the cloud top temperature, a semitransparent pixel is naturally being placed lower in the atmosphere assuming (like the MODIS cloud retrieval does)that it is a fully cloudy pixel. In practice, it means that MODIS may "place" some fraction of "really" high clouds to the middle cloud category. This could also explain the apparent overestimation of high cloud fraction/height by the AIRS cloud retrieval algorithm relative to that of MODIS. Actually, it may even be the main cause behind the fact that the MODIS clouds in each category tend to be at lover levels than the AIRS clouds.

On the other hand, in terms of cloud top pressure, the current AIRS cloud-parameter retrieval algorithm may have difficulties with reliably identifying low clouds, so the average retrieved low cloud top height may shift higher. Nonetheless, the $\mathrm{CO}_{2}$-slicing method employed by MODIS for $\mathrm{C}_{\mathrm{tp}}$ retrieval is also reliable only for clouds above $\sim 700 \mathrm{hPa}$, but the addition of $11 \mu \mathrm{m}$ MODIS window channels greatly improves low cloud detection.

Possible additional reason for differences between MODIS and AIRS Monthly means may be attributed to the fact that the MODIS scan angle is wider than that of AIRS and retrieval accuracy generally decreases with increasing satellite viewing angle, and a cloudy path is also more likely. For Granule-scale intercomparisons this is not a problem, because we could create cloud parameter statistics for coincident grid points only, so comparisons were more 'fair'.

\section{SUMMARY}

- Both the AIRS/MODIS Monthly cloud fractions \& $\mathrm{C}_{\mathrm{tp}} \mathrm{s}$ were highly correlated with each other [0.85-0.90 \& 0.93-0.97, respectively] for both years and both for Aqua \& Terra (higher numbers belong to Terra!). The correlations for inter-annual variabilities were less good; $0.62-0.70$ for Terra, and 0.60-0.64 for Aqua. Note: the correlations between Terra and Aqua cloudiness were only slightly higher; by about 0.03 for cloud fractions and Ctps, and by $\sim 0.06-0.12$ for their inter-annual variabilities. AIRS/MODIS correlations were significantly better when looking at the January vs. August 2004 "seasonal" differences $(\sim 0.80-0.84$ vs. the $0.62-0.70$ exhibited by the inter-annual correlations.

\section{HOWEVER:}

- AIRS low cloud fractions were much lower than those retrieved by MODIS. Most likely explanations were given above, citing the use of thresholds by the MODIS cloud detection/mask/retrieval algorithm $v s$. the "energy balance" type approach of AIRS; The AIRS-retrieved clouds must be fully consistent with forwardcomputed \& measured radiances (e.g., OLR). Currently, there is no such 'sanity check' built into the MODIS cloud retrieval scheme. To further increase the discrepancy, the current AIRS cloud-algorithm may underestimate low clouds simply because of misidentifying some really low clouds and/or fog as surface.

- The cloud top pressures of the AIRS clouds are consistently higher than that of MODIS. This, again, can be largely explained by energy balance considerations; for example, in the longwave spectrum the lower-lying MODIS clouds block less outgoing surface radiation per unit area than the (higher-level) AIRS clouds, so the AIRS clouds must be situated higher (at lower atmospheric temperatures).

- It must be also noted that the MODIS instrument has a much higher spatial resolution than AIRS. Consequently, it can 'see' not just 2 cloud layers/types in an AIRS footprint, but even up to 16 cloud layers (at 4 $\mathrm{km}$ resolution, or even up to 225 cloud layers at $1 \mathrm{~km}$ resolution) in an $\sim(15 \mathrm{~km})^{2}$ AIRS footprint. MODIS to AIRS comparatibility depends crucially how does one average the single cloud parameters referring to a $4 \mathrm{~km}$ (1 $\mathrm{km})^{2}$ pixel. Consequently, cloud type identification can to be more accurate with the well-established MODIS cloud-retrieval algorithm. For now, the AIRS algorithm is not 'tuned' to retrieve thin cirrus, for example, so part of the consistently lower AIRS fractional cloud cover (as compared to MODIS) may be attributable to this. Note, however, that due to good sensitivity of the AIRS algorithm/channels to higher clouds including 'not too thin' cirrus, we are still seeing more high clouds in the AIRS retrievals. 
- These considerations are still preliminary, and other causes may lurk behind the discrepancies between these cloud climatologies. Thus, we are planning to significantly extend the scope of these initial intercomparisons, including comparative assessments using other cloud climatologies (ISCCP and TOVS, for example). All in all, there is not yet a well-established, really "fair" way to compare MODIS to AIRS cloud fractions/cloud top pressures. Our current intercomparisons may provide an initial step to achieve such a goal. For example, comparing both AIRS and MODIS monthly mean cloud statistics to that of high quality ground-based climatologies (ARM sites come to mind), may "decide" which retrieval approaches, if any, may be closer to reality.

\section{REFERENCES}

1. Susskind, J., C. D. Barnet, and J. M. Blaisdell, Retrieval of Atmospheric and Surface Parameters from AIRS/AMSU/HSB Data in the Presence of Clouds. IEEE Trans. Geosci. Remote Sensing, 41, 390-409, February 2003.

2. Zhang, Y., W. B. Rossow, A. A. Lacis, V. Oinas, and M. I. Mishchenko, Calculation of radiative fluxes from the surface to top of atmosphere based on ISCCP and other global data sets: Refinements of the radiative transfer model and the input data, J. Geophy. Res., 109, D19105, 2004.

3. Susskind, J., P. Piraino, L. Rokke,, L. Iredell, and A Mehta, Characteristics of the TOVS Pathfinder A Dataset. Bull Am. Met Soc. 78, 1449-1472, 1997.

4. Pagano, T. S., H. H. Aumann, D. E. Hagan, and K. Overoye, Prelaunch and in-flight radiometric calibration of the Atmospheric Infrared Sounder (AIRS). IEEE Trans. Geosci. Remote Sensing, 41, 265-273, February 2003.

5. King, M. D., Y. J. Kaufman, W. P. Menzel, and D. Tanre, Remote Sensing of Cloud, Aerosol, and Water Vapor Properties from the Moderate Resolution Imaging Spectrometer [MODIS], IEEE Trans. Geosci. and Rem. Sensing, 30, 2-27, 1992.

6. Rossow, W. B., A. W. Walker, and L. C. Garder, Comparison of ISCCP and Other Cloud Amounts. J. Clim. 6, 2394-2415, 1993.

7. Coakley, J. A., Jr., and D. G. Baldwin, Towards the objective analysis of clouds from satellite imagery data, J. Clim. Appl. Meteorol., 23, 1065-1099, 1984.

8. Coakley, J. A., Jr., A Dynamic Threshold Method for Obtaining Cloud Cover from Imagery Data, J. Geophys. Res., 92, 3985-3990, 1987.

9. Wielicki, B. A., L. Parker, and C. Tolson, The Effect of Spatial Resolution on Satellite Derived Cloud Properties, In "Clouds and Climate II," WMO, 1987, 75, 1987.

10. Harshvardhan, B. A. Wielicki, and K. M. Ginger, The Interpretation of Remotely Sensed Cloud Properties from a Model Parameterization Perspective. J. Clim., 7, 1987-1998, 1994.

11. Coakley, J. A., Jr., and F. P. Bretherton, Cloud cover from high-resolution scanner data: Detecting and allowing for partially filled fields of view, J. Geophys. Res., 87, 4917-4932, 1982.

12. Chang, F.-L. and J. A. Coakley, Jr., Estimating Errors in Fractional Cloud Cover Obtained With Infrared Threshold Methods. J. Geophys. Res., 98, 8825-8839, 1993. 
Popular Summary for the paper entitled:

\title{
Validation of AIRS/AMSU cloud retrievals using MODIS cloud analyses
}

\author{
by \\ Gyula I. Molnar and Joel Susskind \\ UMBC/JCET \& NASA GSFC, Code 613, Greenbelt, MD 20771
}

Clouds appear to play an important role in the atmosphere on all spatial and temporal (weather to long-term climate changes) scales. However, our knowledge of their behavior, in particular their interaction with climate changes, is still lacking. Satellites can provide an ideal platform to access cloud and atmospheric variables simultaneously and on comparable scales. This is extremely important for developing more reliable cloud models, which could help to improve the representation of cloudclimate feedbacks (probably the least known ones, still hindering global warming predictions, for example) in global climate models. The TOVS satellites were the first to provide this kind of information since 1979 on. In addition to the retrieval of key atmospheric/surface parameters, Susskind and colleagues have also generated cloud fields using TOVS observations. The recent AIRS/AMSU instrument, continuously operating since September 2002, can accomplish the same tasks with a much higher accuracy. It would be very useful for climate change assessments to have the longest possible satellite cloud climatology. However, so far the AIRS cloud product has not been rigorously evaluated/validated. We describe a validation effort in this paper by comparing AIRS cloud products to MODIS cloud retrievals at varying spatial and temporal scales. In particular, we present statistical differences in the retrieved parameters of cloud fraction and cloud top pressure. We investigate what type of cloud systems are retrieved most consistently with both retrieval schemes, and attempt to assess reasons behind statistically significant differences. Whilst we have found profound differences in the retrieved properties of low-level clouds, for example, depiction of overall cloud variabilities was quite consistent between the AIRS and MODIS schemes, leading hopes that the AIRS cloud data may be useful for assessing potential climatic trends in the retrieved clouds characteristics. 